\title{
REMARKS ON THE DEFORMITY OF PES CAVUS.
}

\author{
BY \\ A. S. BLUNDELL BANKART, F.R.C.S.
}

Pes cavus is a condition in which the arch of the foot is abnormally increased. But, at the outset it is difficult to say what is a normal arch, or rather when exactly a high arch ceases to be normal, because many apparently normal people have high arches, and others have low ones. It is not enough to say that an arch is abnormally high when it is sufficient to give rise to symptoms, because many deformed feet are painless and functionally useful.

The arch of the foot is essentially a dynamic condition, it is an expression of the muscular activity of the foot, and it varies both in different individuals and in the same individual at different times. When the muscles are strongly in action, the arch is well formed or high. When the muscles are relaxed and no weight is put upon the foot, the arch forms a gentle curve representing the anatomical resting position of the skeleton of the foot. When weight is put upon the foot with the muscles relaxed, the arch is depressed even in the normal foot. Thus, changeability, mobility or suppleness, is a feature of the normal arch, and a high arch which cannot flatten is as abnormal as a low arch which cannot be raised.

Pes cavus may, therefore, be defined as a high arch which is fixed, and the symptoms, when they arise, are due not so much to the shape of the foot, as to its lack of intrinsic mobility, its inability to "give" and adapt itself to varying circumstances. In other words, a high arch is abnormal when it ceases to be dynamic and becomes static.

An abnormally high arch is met with as an individual peculiarity in some people who at most complain of difficulty in finding ready-made boots and shoes that are comfortable. It is often associated with short broad hands.

It is said that in some cases a high arch is induced by crowding the feet of growing children into boots and shoes that are too small for them, and that the same effect is produced in women by short narrow shoes and high heels. But, with regard to the latter my experience is quite the reverse; such women nearly always have flat everted feet.

A certain amount of pes cavus is seen in cases of talipes equinus when the patient habitually walks on the toes with the heel off the ground. This occurs both in the normal foot, as when the equinus is merely compensatory for a shortened limb, and in cases of paralytic talipes equinus. In neither case as a rule is the cavus deformity a very prominent feature, and in both it is an almost wholly mechanical effect brought about by compression of the foot in its long axis by the body-weight, and secondary shortening of the soft parts in the sole.

A special place, however, has been given to the slighter degrees of talipes equinus-the so-called right-angled contraction of the tendo Achillisin which the heel is not permanently raised off the ground. In fact, cases of pes cavus have been divided into two groups-those in which dorsiflexion of the foot is limited, and those in which it is not. Here again my experience is that this conditirn (right-angled contraction) is far more often associated 
with flat foot than with pes cavus, and while not denying that some contracture of the sole may occur with the slighter degrees of talipes equinus as well as with the more severe, I would disagree with the view that contraction of the tendo Achillis is particularly associated with the severer grades of pes cavus. In the majority of cases exactly the opposite condition is present-that is, the foot is dorsiflexed at the ankle-joint, while the anterior part of the foot is plantarflexed at the mediotarsal joint. The plantarflexion of the fore part of the foot masks the dorsiflexion at the ankle-joint, and it has been erroneously assumed that because the toes cannot be raised above the level of the heel, the tendo Achillis must be contracted.

Pes cavus then may be associated with other deformities of the foot, but, apart from these there remains a well-defined group of cases in which increase of the arch is the essential condition, and this constitutes pes cavus proper.

The usually accepted explanation of this deformity is that which was given by Duchenne of Boulogne, who attributed it to paralysis of the interossei and lumbricals and of the abductor and flexor brevis hallucis. These muscles flex the toes at the metatarso-phalangeal joints and extend them at the inter-phalangeal joints, so that, when they are paralysed, the toes become hyper-extended at the former and flexed at the latter by the unopposed or uncorrected action of the long extensors and flexors. The proximal phalanges then come to occupy an almost vertical position, and the heads of the metatarsal bones are depressed. Continued action of the long extensors and flexors draws the toes backwards towards the heel, so that the arch of the foot is abnormally increased, and this becomes fixed by contracture of all the soft parts in the sole of the fcot. As the anterior part of the foot becomes depressed in relation to the heel, the patient dorsiflexes the foot at the ankle-joint more and more in order to bring the heel to the ground. But if the deformity progresses beyond the point at which full dorsiflexion of the ankle-joint is reached, the anterior part of the foot then falls below the level to which it is possible to bring the heel, and the patient walks on his toes.

Cases are frequently met with in which there are obvious signs of residual paralysis from a preceding acute poliomyelitis. Pes cavus has also been recorded in cases of injury to the posterior tibial nerve. But, there are many more cases in which it is not possible to demonstrate any paralysis of the muscles, and in which there is no history of paralysis nor indeed of any illness preceding the development of the deformity. It has been suggested that in these cases the paralysis is so slight that it cannot be detected or it is overlooked. But I cannot think that it is right to invent a purely imaginarý illness in order to provide a convenient ætiology for pes cavus. Moreover, there are other features of the deformity which make such an explanation most unlikely.

Pes cavus without evidence of paralysis is quite a characteristic deformity. It is bilateral and it makes its appearance as a rule in the latter part of childhood, usually about the age of seven to ten, when without apparent cause the sole of the foot becomes slightly hollowed out and the toes show a tendency to become clawed. The deformity slowly, but surely, increases, the sole becoming more and more concave, and the dorsum of the foot unduly prominent and convex, while the toes are strongly hyperextended at the metatarso-phalangeal joints and flexed at the interphalangeal joints. The weight of the body is then borne entirely on the heel and the heads of the metatarsal bones, the latter forming a prominent "ball " of the foot. Painful corns and callosities are common beneath the heads of the metatarsals and over the proximal interphalangeal joints, where they press 
against the boot. The gait is awkward, stumpy and inelastic, and the patient may suffer much from the painful callosities and corns. Voluntary movements of the foot and toes appear to be normal except in so far as they are limited by the fixed deformity. No evidence of actual paralysis can be found clinically, though it must be admitted that isolated paralysis of the small muscles is difficult, if not impossible, to demonstrate in a contracted foot.

This deformity then comes on gradually and without apparent cause at a more or less definite age. It is in many cases hereditary, and several members of the same family may be affected. When two or more children in a family are affected, they do not develop the deformity simultaneously, but each child begins to show signs of it as he reaches the characteristic age of onset.

These features strongly suggest some congenital defect, rather than an accidental infection, as the underlying cause of pes cavus. It is perhaps significant that some of the most typical examples of this deformity ocqur in association with Friedreich's ataxy-a condition which is itself hereditary and familial and which is generally considered to be of developmental origin. It should be specially noted that none of the characteristic spinal cord degenerations of Friedreich's ataxy is responsible for a peripheral paralysis such as could give rise to pes cavus, and that the clinical picture is that of associated developmental defects, rather than one of primary cord disease leading to secondary paralytic deformity.

The condition invites comparison with the atrophic claw hand which may be due to a cervical rib. In this case a congenital anomaly lies latent until adolescent or adult age, when the altered relationship between the rib and the surrounding parts gradually causes pressure on the lower roots of the brachial plexus, with consequent atrophy of the intrinsic muscles of the hand. Even more suggestive is the fact that a similar paralysis may occasionally be brought about by pressure between the brachial plexus and a normally situated first rib. Whether this is associated particularly with a postfixed plexus, I do not know; but clearly there must be some developmental fault in the relationship of the plexus to the rib.

-During childhood a very considerable alteration occurs in the relations between the spinal cord and the vertebral column. Extending originally the whole length of the spinal canal, the cord at birth ends opposite the body of the third lumbar vertebra, while in the adult it reaches only to the lower border of the body of the first lumbar vertebra or the upper border of the second. In this process of relative shortening the lumbosacral nerve roots become drawn out and obliquely placed between their attachments to the cord and their exit from the vertebral foramina. Variations in the position and constitution of the lumbosacral plexus are frequent and are commonly associated .with variations in the vertebral column. It is not difficult to imagine that slight developmental defects in the lumbosacral region of the spine or in the costal elements of the sacrum might give rise to pressure on the roots of the sacral plexus comparable to that which occasionally occurs in the cervical region. These considerations have led one to search for evidence of developmental anomalies in the neighbourhood of the first sacral nerve roots in cases of pes cavus, both with and without Friedreich's ataxy.

-NaturaHy one looks first for radiographic evidence, and many cases of apparent defects have been noted in these cases, e.g., spina bifida occulta; sacralisation or partial sacralisation of the fifth lumbar vertebra, while others again were apparently normal. But, the radiographic appearances in this 
region are so variable and such fanciful interpretations have sometimes been given to them, that one hesitates to draw any conclusions from these cases.

In three cases I have explored the region of the first sacral roots. In one case there was a defect in the lamina of the fifth lumbar vertebra, in another the first sacral roots appeared to be smaller than normal, while in the third there was a definite thickening of the dura mater at the level of the fifth lumbar and first sacral vertebræ. It has not, however, been possible to draw any definite conclusions from these cases and I have had no opportunity of examining a case of pes cavus post mortem.

\section{Treatment.}

The treatment of the deformity of pes cavus has been much simplified by the introduction of Steindler's operation of " stripping the os calcis." Formerly, these cases were treated by subcutaneous division of the contracted fascia in the sole, and wrenching and manipulations and splints, and while there is no doubt that the deformity in most cases was corrected by these means, the general experience was that they showed a very great tendency to relapse. Steindler's operation aims at detaching all the soft parts from the posterior pillar of the arch of the foot, namely, the os calcis, so that when the foot is straightened, they slide forward and gain a new attachment further forward. This principle of shifting the attachments of contracted soft parts, rather than dividing them in the middle and allowing them to heal by scar tissue, is a great advance in the correction of deformity, and cases so treated do not show nearly the same tendency to relapse. Pes cavus therefore can in most cases be corrected without difficulty, and the comfort of the patient is greatly increased thereby. 